\title{
The replication crisis, the rise of new research practices and what it means for experimental economics
}

ESA Report

Lionel Page ${ }^{*}$

Charles N. Noussair

Robert Slonim

\section{Introduction}

In the wake of the replication crisis in psychology, a range of new approaches has been advocated to improve scientific practices and the replicability of published studies in the behavioural sciences. The ESA Executive Committee commissioned an ad hoc committee to review the issues raised by the replication crisis, and how they affect research in experimental economics. The present report is the result of this review. Its content has greatly benefited from the personal views and insights of a large number of ESA members. The views within the community of researchers in experimental economics are diverse. The present report does not aim at determining a strict ESA policy. Rather, it aims to bring to the community of experimental economists the collective wisdom spread among experimentalists. The report presents a discussion of the different issues related to replicability and discusses the different potential solutions, with their benefits and pitfalls. The report also contains a series of recommendations that aim to address the challenges presented by the replication crisis, while respecting the diversity of views within the ESA community.

\section{The issues raised by the replication crisis}

In recent years, there has been a growing concern that, for a substantial proportion of studies published in scientific journals, the results are false (Ioannidis, 2005). This concern has been particularly strong in psychology, and it has spread to other social sciences, including economics. In psychology, many studies, some of which are very well-known and discussed in textbooks, have seen attempts at replication fail. In some cases, whole areas of established research have been cast into doubt.

These facts have prompted a discussion about the causes of these replicability failures. It is now commonly agreed that a large part of the issue arises from the structure of incentives in the process of producing and publishing scientific results. Science is a human practice, and researchers and editors have incentives, which may, in some instances, favour outcomes at odds with the ideal process of scientific discovery. The replication crisis can be seen as a consequence of these incentives and their implications for the use of questionable research and publication practices.

\footnotetext{
* Page: University of Technology Sydney, Sydney, NSW, Australia. Noussair: Department of Economics, University of Arizona, Tucson, AZ, USA. Slonim: University of Technology Sydney, Sydney, NSW, Australia.
} 
Authors have an incentive to publish in high impact journals; and editors have an incentive to publish findings which are cited more (to raise measures of the impact of their journal). Statistically significant findings are more likely to be cited than null results (Duyx et al. 2017). To some extent, it can also be justified for editors to select significant findings in order to inform policy (Kasy, 2021). As a consequence, editors have an incentive to publish significant findings and authors have an incentive to find significant findings. The rewards from publishing statistically significant findings lead to distorting incentives to find something significant to publish. It leads to biases in the publication of scientific results which ideally should be about publishing results that answer interesting questions, regardless of whether or not they yield significant or null results.

Several mechanisms have been identified as likely drivers of the publication of too many false positive results.

\subsection{File-Drawer effect: Not enough incentives to publish null results.}

The first mechanism is the file-drawer effect. This term refers to the fact that studies with null results are harder to publish. Authors anticipate this and often do not write up non-significant results. These results end up staying in the researcher's drawer: they are never published.

This effect has several negative consequences. One of them is the wasteful repetition of unsuccessful studies, because previously failed attempts are not publicly recorded. Another is a misleading body of reported findings in which variables are thought to have stronger relationships than is justified. The file-drawer effect can also take place within studies. A research team may run several studies linked to each other and only publish the studies/questions that yield significant results, while leaving unwritten the studies/questions that did not.

\subsection{P-hacking: Incentives to mine the data for significant relationships.}

The second mechanism is the practice of $p$-hacking. This term refers to the fact that researchers have an incentive to use the many degrees of freedom in the available data analyses techniques to find one type of analysis (model, variables, sample), which delivers a significant result.

In the case of experiments, a possible form of p-hacking is the systematic investigation of subsamples. When researchers do not find a significant main effect for their experimental treatment, there is an incentive to look for effects in sub-samples. If researchers were not looking for such effects in sub-samples in the first place, the study's design may not be powered to do so. Besides, researchers may carry tests on many sub-samples and report only the subsamples for which the result is significant. Doing so, they may fail to correct for multiple hypothesis testing. In the end some significant results arising from these analyses are presented without providing a full picture of the whole set of tests that have been carried out. This practice leads to the publication of many false positive results, since the probability of getting at least one significant test among many tests is much larger than the critical p-value of the test (e.g. 5\%).

\subsection{Harking: Incentives to build narratives fitting whatever results are found ex-post.}

A third mechanism is the practice of harking. This term comes from the fact that, having found something significant from multiple testing, researchers can reverse engineer "hypotheses" after the results are known (HARK). The unexpected results are then presented as reflecting hypotheses which were meant to be investigated in the first place. 


\subsection{How much of a problem is it for experimental economics?}

Compared to other research areas, laboratory experiments have fared relatively well in the wake of the replication crisis (Camerer et al. 2016). There are possibly several reasons for this. One possibility is the existence in economics of a shared theoretical framework, which de facto constrains the type of hypotheses that can be generated to explain results after the fact. Another possible contributing factor is that hypothesis testing is likely more expensive in economics where experiments are incentivised. The possible practice of running many experiments to study many questions, in order to select only a few to focus on ex-post, is less likely to happen in experimental economics than in other disciplines, like psychology, where collecting experimental data is cheaper. Experimental economics is, however, not immune to the concerns raised by the replicability crisis.

Considering p-hacking, Brodeur et al. (2016) provide interesting evidence on its prevalence in economics and its sub-disciplines. The practice of $p$-hacking can be detected among many published studies by the bunching of test statistics just above the significance level necessary for results to be significant. Figure 1, taken from their published paper, shows that experimental papers display less bunching of test statistics just above the $5 \%$ threshold (z-statistic equal to 1.96). Instead, in other areas in economics, there is a clear peak just above z-statistics of 1.96.
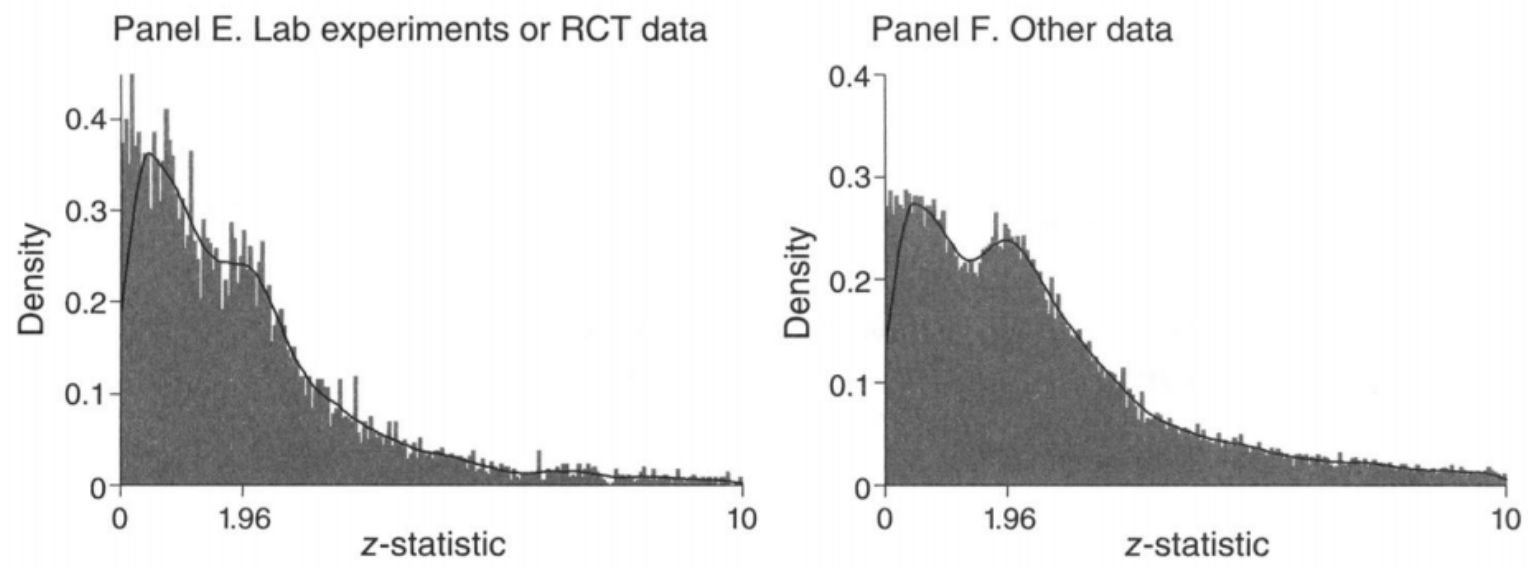

Figure 1: distribution of z-statistics in experimental versus non-experimental studies. ${ }^{1}$

The published version of this figure lumps laboratory and field experiments together. The working paper version, Brodeur et al. (2013), gives a slightly less positive picture for (laboratory) experimental economics than for randomized control trials (RCTs). In spite of the noisier data (likely due to the lower number of observations), there seems to be barely any second peak in RCTs, while the lab experiment distribution seems closer to the non-experimental data. A possible explanation is that RCTs may be less affected by $p$-hacking because, given the size and cost of the studies, authors push more to publish results, even when they are null.

\footnotetext{
${ }^{1}$ Copyright American Economic Association; reproduced with permission of the American Economic Journal: Applied Economics
} 


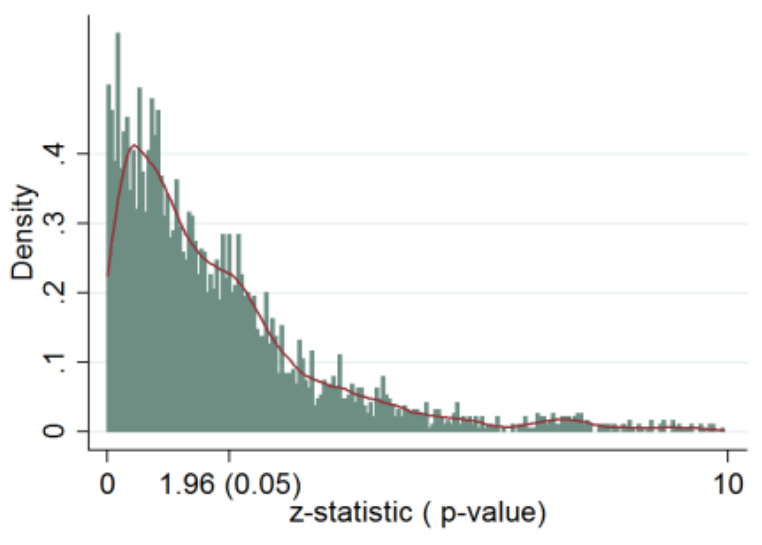

(a) Randomized control trials data.

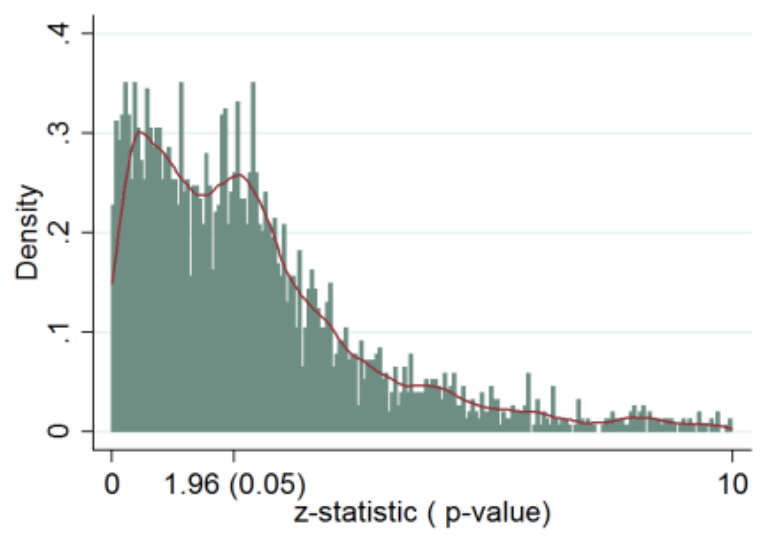

(b) Laboratory experiments data.

Figure 2: distribution of z-statistics in Randomized Control Trials versus Laboratory studies. ${ }^{2}$

Another piece of evidence is provided by Figure 3, taken from Kasy (2021). It compares the normalised estimates from 18 laboratory experiments published in top economics journals in the years 2011-2014 and the normalised estimates of their replications by Camerer et al. (2016). For the original estimates, we observe a bunching of estimates just above 2 , the threshold associated with statistical significance at $5 \%$. This pattern suggests both that non-significant findings are less likely to be published and that some of the significant estimates may stem from authors' degrees of freedom to find significant results. Most importantly, we also observe that most of these marginally significant estimates dropped below 2 in replications, indicating a failure to replicate.

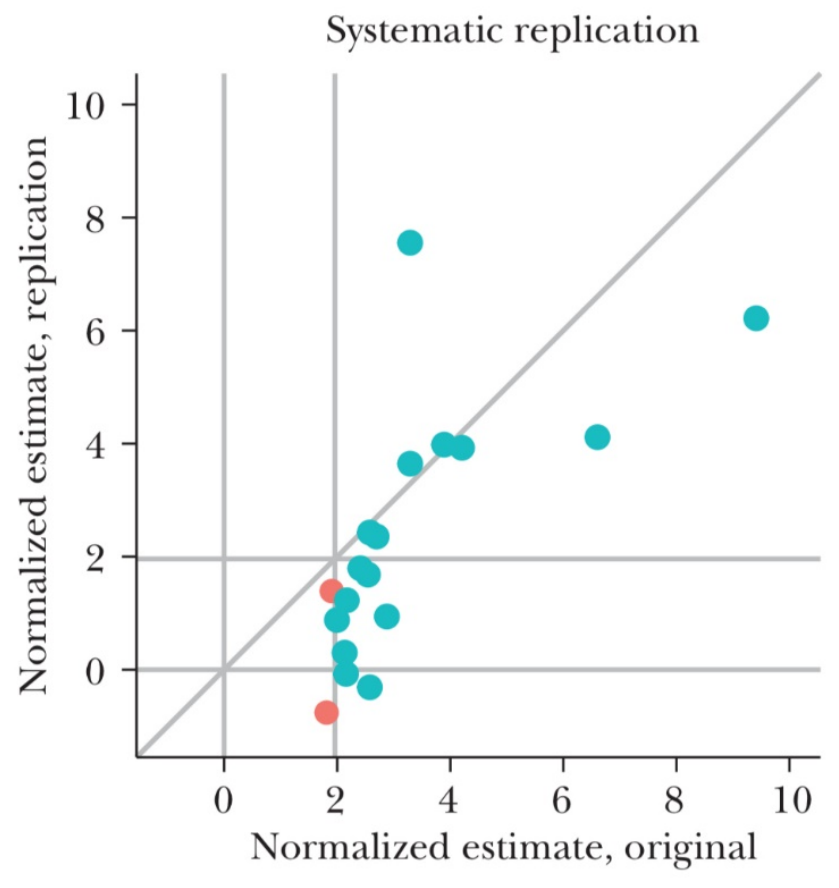

Figure 3: comparison of normalised estimates between original and replicated studies ${ }^{3}$

\footnotetext{
${ }^{2}$ Reproduced with permission of Abel Brodeur.

${ }^{3}$ Reproduced with permission of Maximilian Kasy
} 
Experimental economics is also not immune to the issues raised by the practice of harking. In particular, many experimental papers are increasingly motivated by conjectures that are not driven from economic theory. It is likely a result of the relative decline of the primacy of classical economic theory and the greater openness to ideas coming from other social sciences. Researchers are now able to justify their research with 'reasonable' assumptions and conjectures reflecting insights from other disciplines such as psychology or sociology where many different opposing hypotheses and views can co-exist. In addition, within economics there has also been an increasing number of alternative theories, for instance in the area of social preferences. This greater variety of possible theories and empirical conjectures offers increasing options for hypothesis testing. As a consequence, experimental economics is not immune to researchers having many degrees of freedom when designing hypotheses to fit the data. It is especially the case when there is little existing precise theory disciplining the design and a lot of data is being gathered, such as studies that include extensive psychological measures or physiological data.

\section{Solutions}

In the wake of the replicability crisis, many new practices and solutions have emerged (for a review see Christensen and Miguel, 2018). There is not full consensus among experimentalists on these practices still they have affected the discipline in significant ways. We review here some of the main solutions proposed to improve scientific practices and lead to a greater replicability of published studies.

\subsection{Pre-registration}

The pre-registration of hypotheses has been proposed as one of the tools to improve research practices by reducing researchers' freedom to engage in harking and $p$-hacking. We discuss the preregistration of hypotheses and the pre-registration of an analysis plan separately.

\subsubsection{Pre-registration of hypotheses}

The pre-registration of hypotheses requires publishing, on a third-party platform, a document describing the intended study and the hypotheses tested. Part or all of the document may be kept private until publication.

Pros

Drawer effect: By providing a record of the study attempted, pre-registration limits the drawer effect. It gives visibility to previous attempts at answering a question.

P-hacking: By specifying the main hypotheses beforehand, pre-registration prevents the researcher from fishing for significant results through multiple testing over different sub-samples, when the hypothesized result is not found. A pre-registration implies that such tests can still be carried out, but they have to be explicitly presented as exploratory, ideally with the whole set of tests conducted being presented.

Harking: The pre-registration of hypotheses limits harking. It puts on record what were the effects that were expected/investigated. Results that are surprising are more credible when hypotheses are pre-registered. Effects which are found but were not pre-registered have lower credibility in comparison. Surprising hypotheses which are not pre-registered can be suspected to be ex post rationalisations of results. Such results have a high chance of being false positives.

\section{Cons}

The pre-registration of hypotheses may not deliver the intended benefits. 
Pre-registration may reduce the drawer effect, but it is unlikely to eliminate it. Even pre-registered, null effects are still less likely to be published or even written up. They are therefore less likely to be included in the public record.

Pre-registration creates an additional cost in the process of conducting and publishing a scientific study. Even if some of the pre-registration work consists merely of frontloading the conceptual work for an experiment, it still adds some administrative time. It involves writing a document which needs to respect some specific format constraints. It may potentially create some inequality between researchers in large laboratories with junior researchers/research assistants (who often carry the bulk of the administrative work) and researchers in smaller research units (who have the whole responsibility for the design and running of their experiments).

The pre-registration of hypotheses also raises the issue of making scientific ideas public before publication. There are two main concerns:

First, ideas could be scooped if the pre-registration is public. One solution is not to require that the preregistered hypotheses are made public. In that case, it could only be required for the editor and reviewers to have access to it when the paper is submitted to a journal. The pre-registration can be made public when the paper is published.

Second, there could be a risk of "pre-registration trolling" in a way similar to patent trolling. A research group could potentially pre-register a large number of ideas to lock intellectual property rights on them without the intent to work on all of them. The payout could be to have the opportunity to select promising projects at a later date as a function of the evolution of the field. One could even envisage that other researchers would offer to co-author these pre-registered studies and carry out the work, in effect paying a rent to the researchers who pre-registered many ideas. While we are not aware of any reports of such practices, it is important to anticipate the types of incentives which could arise from the widespread use of pre-registration. The concern about possible pre-registration trolling raises some questions about the notion that pre-registrations should be seen as establishing property rights. These hazards can be eliminated by making preregistration private.

\subsubsection{Pre-registered analysis plans}

The pre-registration of an entire analysis plan goes a step further than the pre-registration of hypotheses. It requires the registration of the statistical analysis that is to be used to analyze the experiment data. While often conflated with pre-registration, it is often not required in the preregistration process. It is, for instance, not required by the AEA RCT registry. A pre-registration with analysis plan can, therefore, be described in short as the pre-registration-plus option.

\section{Pros}

Planning. The first merit of the pre-analysis plan may be to force researchers to think more carefully ex-ante about their intended analyses and therefore, their design. There is potential to improve the quality of experiments being run. In particular, in terms of the important issue of achieving adequate statistical power, pre-analysis plans may foster better practices, with larger samples acquired when needed.

P-hacking. The second merit of the pre-registration of an analysis plan is to limit p-hacking. By clearly specifying the analyses prior to the study, the analysis plan acts as a pre-commitment tool which limits the degrees of freedom of the researchers in the analysis process. As a consequence, it increases the credibility of the results derived from pre-registered analyses. New analyses that are 
not pre-registered are still possible. The pre-registered analysis plan simply makes it clear that these analyses are exploratory and have, by design, a lower degree of strength as evidence.

\section{Cons}

Coffman and Niederle (JEP, 2015) pointed out that analyses plans may not dramatically decrease false positives, if there are many competing studies being conducted (either by the same researchers/labs or by different researchers). Intuitively, if 20 analysis plans are written up, then on average one will deliver a significant result under the null. If publications still favour significant results, published results will still overly feature false positives.

There are also risks that pre-analysis plans may constrain the researchers when investigating empirical results. A risk is that "exploratory analyses" end up being too devalued or dismissed by reviewers and editors. The emergence of such a convention could be detrimental.

In terms of methods, it is dubious to think that there is no insight to gain from looking at the data to determine the best way to analyse it (Olken, 2015). For a start, researchers often realise that the analyses they initially planned were ill-conceived, or at least not perfectly conceived, after beginning to run the experimental sessions or when looking at the data. One reason for this is that it can be difficult to adequately anticipate all the aspects and challenges that characterise a dataset before working on it, especially if it is a novel type of experiment.

Regarding the results, requiring a strict pre-analysis plan to consider a result as worthy of being credible would prevent researchers from publishing results they did not expect. This does not seem to be a reasonable approach.

We believe that the view of Simmons et al. (2018) should prevail: "Pre-registration does not restrict the ability to conduct exploratory analyses; it merely allows the researcher and the reader to properly distinguish between analyses that were planned vs. exploratory."

\subsection{Registered reports}

Registered reports consist of moving the publication point one step back and allowing researchers to submit their pre-registration for consideration for publication. If the registered report is accepted, it seems in practice to work as a strong R\&R with high chances of publication for the final study (if it does not deviate too far from the accepted registration).

\section{Pros:}

Drawer effect. Registered reports have the potential to limit the publication bias on both the editorial and the author side. Editors and reviewers have to decide on the interest of the proposed study before any data is gathered. As editors have a clear incentive to publish significant results, registered reports offer an interesting solution to this problem. On the other hand, editors may still accept papers based on their beliefs about what the results might turn out to be, though this is not an easy task in many instances.

Decision time. An additional benefit of registered reports is that the acceptance decision is frontloaded earlier in the research project. This timing is better for researchers, in particular, earlycareer researchers who need evidence of publication success when going on the job market or being considered for promotion or tenure.

In terms of effort, pre-registered reports could in some cases decrease the time spent writing papers. It is not uncommon for authors to re-write papers along different narratives as they 
progressively investigate their data further. One of these scenarios is when an early working paper with null results becomes a published paper with significant results on a subgroup of the participants or a change in focus to a secondary question. Registered reports force the results to only be written once with a given angle, and this angle is the only aspect of the paper that needs to be addressed with the data once it is collected.

\section{Cons}

There are, in theory, possible risks of anti-selection and moral hazard:

Anti-selection: researchers could potentially run several studies and submit as pre-registered reports only those that are not significant. This would contravene the intent of such reports to be registered before any data is collected.

\section{Moral hazard:}

1. Researchers could decrease their effort after getting an acceptance so that the quality of the papers published is lower.

2. Researchers could withdraw their paper after acceptance if the results turn out to be very appealing and submit it to a higher-ranked journal. They could even send a paper after acceptance to a higher-ranked journal and then back to the initial journal which offered a registered report if it did not work out at the higher journal.

It seems to us that these risks are not too high given the reputational costs researchers could face. But editors should be aware of them when designing rules for making acceptance decisions for registered reports.

An interesting alternative is the idea to submit papers of fully finished studies but where the results are masked. Such so-called "sealed envelope submissions" would induce the editors and reviewers to make their evaluation of the paper's quality and contribution based on the method and on the question rather than based on the results. Martin Dufwenberg and Peter Martinsson (2014), who proposed this solution, stress that it would reduce the incentives of the researchers to $p$-hack. This option is appealing by being less costly for the researchers than writing a pre-registered report prior to the study. There is obviously a risk of anti-selection, whereby a journal offering this option could receive a disproportionate number of studies with null results. There is also a question of credibility in preventing information about the results (which already exist) from reaching the editors and referees. Working papers are frequently online, which makes it easy to find the results. Papers are also often presented prior to submission, which may lead to information leakage.

\subsection{Replications}

Replications are attempts to reproduce published results. We can distinguish exact replications (different team, same method) and conceptual replications (different team, different method). We consider here primarily replications of laboratory and online experimental studies, not RCTs in the field which, by their nature (size, expense, and specific sample requirements) are harder to replicate.

Pros:

It is widely accepted that replications are highly desirable. They confirm or contradict previous results and help to consolidate the set of accepted results in a field.

\section{Cons}


Replications are not free of problems. One possible issue is that the promotion of replications may distort incentives in another way. Replications may be more likely to be published when their results contradict the original studies and are therefore surprising. This publication bias may lead researchers overly focusing on disproving past results.

Another problem with replications is that they are often not visible enough. Many so-called zombie results may survive a long time in the collective beliefs of a scientific community while their reality has been undermined by failed replications. This fact also contributes to an asymmetry of incentives to produce good research. Anecdotal evidence suggests that it is much more rewarding for a researcher's career to publish a paper with invalid results in a top economic journal than to publish a rigorous replication in another outlet that overturns a result that appeared in a top journal.

\section{Recommendations}

Table 1 summarises the different problems and solutions that we have discussed here with their pros and cons.

\begin{tabular}{|l|l|l|l|l|l|}
\hline & $\begin{array}{l}\text { File } \\
\text { drawer }\end{array}$ & P-hacking & Harking & \multicolumn{2}{|c|}{ Overall } \\
\hline & Addresses & Addresses & Addresses & Pros & Cons \\
\hline $\begin{array}{l}\text { Pre- } \\
\text { registrations }\end{array}$ & $\checkmark$ & $\checkmark$ & $\checkmark$ & $\begin{array}{l}\text { Public record, Pre-commitment to } \\
\text { hypotheses and analyses }\end{array}$ & $\begin{array}{l}\text { Costs, gains may be limited in some } \\
\text { cases, risk of seeing exploratory } \\
\text { analyses not published, risk of trolling }\end{array}$ \\
\hline $\begin{array}{l}\text { Registered } \\
\text { reports }\end{array}$ & $\checkmark$ & $\checkmark$ & $\checkmark$ & $\begin{array}{l}\text { Pre-commitment to hypotheses } \\
\text { and analyses, decision time }\end{array}$ & $\begin{array}{l}\text { Negative selection of studies, less } \\
\text { motivation to write the final paper }\end{array}$ \\
\hline Replications & & $\checkmark$ & $\checkmark$ & Ensure robustness of results & Risk of incentives to disprove results \\
\hline
\end{tabular}

Table 1: The different issues identified as contributing to the replicability crisis and how the different solutions help address them.

The replication crisis raises important questions for the community of experimental economists. Several innovations to the process of publishing scientific studies have been proposed and they deserve the attention of the community of experimental economists. Our view on the different solutions proposed and the way to use them are summarised as follows:

\subsection{Pre-registration}

Pre-registration can be useful and it would be worth promoting its use. Our view is that preregistration should not be imposed as a blanket condition for publication in experimental economics. Letting the academic norms progress with an encouragement for the practice of pre-registration and a monitoring of possible unexpected consequences seems to us the right way forward. The following encouragement can be considered:

Researchers: The decisions to (1) pre-register and (2) register hypotheses only or analyses as well can be presented as a choice by journals. Pre-registered hypotheses and analyses would have stronger credibility in the review process, especially in cases such as (but not limited to) theory testing and replication studies, where the hypotheses may fall directly out of a theoretical model or the findings of the previous study that is being replicated. But pre-registering hypotheses may be less relevant in situations where a question/design is novel and more exploratory. In such cases, the researchers' priors may not point to precise hypotheses and exploratory analyses may make more sense than artificially pre-committing to some hypotheses. With that in mind, it may be better for researchers to be free to choose whether or not to register some or all hypotheses, given the tradeoffs they face in their specific inquiry. On the other hand, in such studies there is greater scope for 
harking, and researchers can benefit greatly if they are able to pre-register the hypotheses that they do have in order to convince the reader that harking is not occurring. Pre-registration could be private, but the files should be accessible to reviewers and editors during the submission process.

Editors, Reviewers: Pre-registrations should not preclude the publication of exploratory analyses. Researchers should still be welcome to submit exploratory results, with possibly a more conservative way of appraising them. For instance, greater statistical significance may be required to establish confidence in such results. Alternatively, results may be presented as suggestive and invitations for further research. One possibility would be for editors to propose a standard format for published papers in which tests of hypotheses and exploratory analyses are reported in two different sections of the paper. Another option available to editors, when faced with exploratory results that are quite unexpected, could be to request that some parts of the study be rerun to ascertain the robustness of the results. This approach cannot be used all the time given the cost of rerunning studies, but in the case of laboratory experiments, rerunning sessions is feasible in the revision process. With such solutions in mind, we believe that, if pre-registrations become more common, editors and reviewers should refrain from discouraging researchers from publishing exploratory results per se.

In order to help the reviewing process, while also limiting concerns about the theft of intellectual property, pre-registration can be private and only available to reviewers/editors until publication of the research study. The pre-registration can be made public after the study is accepted for publication. The choice to make a pre-registration private could be left to the authors.

\subsection{Registered reports}

Registered reports are a new and interesting avenue for scientific publications. At the moment, only Nature Human Behaviour (NHB) has a registered report section for experimental papers. At the time of this writing, Experimental Economics is currently putting together a symposium trialling this new type of publication. The discussions in the ESA around this document revealed some divergence of views about the merits of this approach. The ESA may explore how its journals could allow such a publishing option, with a plan to assess its success.

The use of registered reports would need to be carefully implemented, however. NHB has adopted a registered report option, but it has put the bar much higher in terms of requirements than for its normal paper section, presumably to counter anti-selection. It may, possibly, make it much harder to publish an experimental paper there than through the normal process.

Given that the registration can either consist of hypotheses only or of hypotheses plus analysis, one could, in the same way, consider two different types of registered reports:

Editorial decision based on the registration of hypotheses and design (=pre-submission enquiry+). Researchers propose a design and hypotheses and get an early indication of interest from editors and potential referees.

Editorial decision based on full pre-registration (perhaps including the analysis plan). This is what standard registered reports are.

The editorial commitment following the initial editorial decision for the first type would be weaker than for the second type of pre-registration.

\subsection{Replications}

Replications are highly desirable for scientific advancement, but currently do not provide enough of an incentive for most researchers to conduct them. At the moment, the incentives are not present 
for editors or authors to invest a lot of effort in replications. Editors want to publish novel results, since scientific recognition comes from innovative ideas, and therefore replications are less rewarded and less welcome in leading journals. Even if published, a successful replication would validate the citation of the initial study. It would therefore typically not attract many citations on its own, unless a norm develops for replications to be cited along with the original study. We would encourage such a norm. Researchers therefore have more incentives to produce original research than to work on replications.

To induce more replications, changing incentives is therefore likely required. Through its journal, JESA, ESA is already encouraging the publication of replications, although with a moderate success despite the relatively high acceptance rate for these. So far JESA has received only 21 such submissions, 7 of the 18 that have gone through the editorial process were accepted, implying an acceptance rate of $39 \%$, higher than the usual acceptance rate for JESA, which is below $30 \%$.

Any recommendation has to face the fact that proposals to change behaviour are unlikely to be effective when incentives are stacked against replications. Solutions have to consider changing the incentives. For instance: journals could consider having special issues dedicated to replications. It would allocate some predetermined space to replications without editorial decisions putting them in competition with non-replication studies, and a prize could be awarded to the best replication study. Replications can also make a natural training exercise for PhD students. Having avenues to publish replication studies could provide the needed incentive for them to do replications. The size of replications is important, and the ESA may have a role in fostering multi-lab collaborations to replicate findings.

Replications often have an underwhelming effect on the ability of a research field to self-correct by abandoning zombie results. Here again, improving this situation likely requires a change of incentives. One possible goal would be to decrease the lifetime of zombie results by improving the dissemination of information on failed replications.

A critical time when researchers need to be aware of existing replications is when they review the literature on a topic. When considering a specific reference, they would benefit from having quick access to the papers replicating the study. At the moment, finding replications is time consuming. Using Google Scholar, for instance, an author would have to browse many papers to find replications. Ideally, Google Scholar would include a link below each paper to "Replication" studies. Replication studies would reproduce the result with different researchers but the same experimental set up. Another possible option could be for a Replicability repository platform (e.g. a wiki) to emerge. This platform could be filled by researchers themselves. Researchers running a replication would have the incentive to create a record for their target paper with a list of replications of the original result, including their own study.

A public platform listing replications could also serve as a metric to judge the quality of a paper. Authors could then list their papers with their citations and the number of successful replications on their CVs. The idea of using replications as a metric of quality was suggested by Maniadis, Tufano and List (2015).

Maniadis, Tufano and List (2015) also considered the incentives of the original author to facilitate replication. They suggested an editorial policy of always allowing original authors to comment on replications. We envision that the replication and response would often occur in a journal other than the one in which the initial study was published. It has automatic benefits in terms of publications 
and citations, encouraging and rewarding discussions on research topics. The space required for comments can be small and therefore not be too costly for journals.

Another idea could be for the journals to publish, along with all the other statistics they report, how many of their experimental studies have had new studies that replicate or have failed to replicate their results in the past year. For instance: " 22 new papers appeared in 2021 that included replications of 17 papers published in our journal. Of these 22 papers, 20 replicated the original results while 2 failed to replicate the original results."

The journals could link to the list of replications. These would be relevant to give exposure to the replication studies and would contribute to increasing the incentives of researchers to produce replications (as well as the incentives of editors to publish studies which are going to be replicated).

\section{Conclusion}

In this report, we have aimed at helping the community of experimental economists-researchers, reviewers and editors - to have a common understanding of the evolutions in scientific practices taking place following the replicability crisis. We have also aimed to give suggestions and recommendations reflecting the views of the ESA community on how practices in experimental economics should evolve.

Scientific practices evolve over time. Scientific norms can be conceived as equilibria and it is often hard to predict beforehand all the consequences resulting from establishing new norms. Our goal with our recommendations has been to point to ideas and suggestions which are fairly consensual among experimental economists because the benefits are perceived to clearly outweigh the costs. By making these recommendations public in the Journal of the Economic Science Association, this report can help researchers reach a common understanding of what is seen as desirable or expected in the field. At the same time, we have been careful to point to the uncertainties associated with the solutions being discussed. We are mindful that, on several issues, the opinions are not consensual within the ESA community, in large part because different members do not foresee the same balance of expected costs and benefits. We are also mindful of the fact that it is hard to anticipate the many ways in which researchers', reviewers' and editors' decisions will change as a result of new scientific practices. These considerations have motivated our characterisation of our recommendations as suggestions to help shape the evolution of the norms in experimental economics rather than injunctions to be implemented as rules.

We are confident that the state of the research in experimental economics is relatively healthy, even if we do not shy away from acknowledging that experimental economics is not shielded from the concerns which have arisen from the replicability crisis. We are also confident that the community of experimental economists can greatly benefit from adapting and adopting some of the solutions which have emerged to the replicability crisis to further improve the quality of the research produced in experimental economics.

Aknowledgements. This report benefited from comments, feedback and suggestions from a large number of ESA members. Among those, we would like to thank particularly Edward Cartwright, Noemi Peter, Alex Roomets, Egon Tripodi, Irenaeus Wolff, and the JESA editors Maria Bigoni and Dirk Engelmann. 


\section{$\underline{\text { References }}$}

Brodeur, A., Lé, M., Sangnier, M. and Zylberberg, Y., 2013. Star Wars: The empirics strike back (No. 7268). IZA Discussion Papers.

Brodeur, A., Lé, M., Sangnier, M. and Zylberberg, Y., 2016. Star wars: The empirics strike back. American Economic Journal: Applied Economics, 8(1), pp.1-32.

Camerer, C.F., Dreber, A., Forsell, E., Ho, T.H., Huber, J., Johannesson, M., Kirchler, M., Almenberg, J., Altmejd, A., Chan, T. and Heikensten, E., 2016. Evaluating replicability of laboratory experiments in economics. Science, 351(6280), pp.1433-1436.

Christensen, G. and Miguel, E., 2018. Transparency, reproducibility, and the credibility of economics research. Journal of Economic Literature, 56(3), pp.920-80.

Coffman, L.C. and Niederle, M., 2015. Pre-analysis plans have limited upside, especially where replications are feasible. Journal of Economic Perspectives, 29(3), pp.81-98.

Dufwenberg, M. and Martinsson, P., 2014. Keeping researchers honest: The case for sealed-envelopesubmissions. IGIER (Innocenzo Gasparini Institute for Economic Research), (533).

Duyx, B., Urlings, M.J., Swaen, G.M., Bouter, L.M. and Zeegers, M.P., 2017. Scientific citations favor positive results: a systematic review and meta-analysis. Journal of clinical epidemiology, 88, pp.92-101.

loannidis, J.P., 2005. Why most published research findings are false. PLoS medicine, 2(8), p.e124.

Kasy, M., 2021. Of forking paths and tied hands: Selective publication of findings, and what economists should do about it. Journal of Economic Perspectives, 35(3), pp.175-92.

Maniadis, Z., Tufano, F. and List, J.A., 2015. How to make experimental economics research more reproducible: Lessons from other disciplines and a new proposal. In Replication in experimental economics. Emerald Group Publishing Limited.

Olken, B.A., 2015. Promises and perils of pre-analysis plans. Journal of Economic Perspectives, 29(3), pp.61-80.

Simmons, J.P., Nelson, L.D. and Simonsohn, U., 2018. False-positive citations. Perspectives on Psychological Science, 13(2), pp.255-259. 